Polymer Journal, Vol. 39, No. 6, pp. 568-578 (2007)

(C) 2007 The Society of Polymer Science, Japan

\title{
Structural Changes of Polyacrylonitrile Film by Catalytic Effects of Nickel Particles under Carbonization Process
}

\author{
Qingyun Chen, Xiaowen Jiang, Yuezhen Bin, and Masaru MATSuo ${ }^{\dagger}$ \\ Department of Textile and Apparel Science, Faculty of Human Life and Environment, \\ Nara Women's University, Nara 630-8506, Japan
}

(Received December 18, 2006; Accepted March 9, 2007; Published April 23, 2007)

\begin{abstract}
Structural changes of polyacrylonitrile (PAN) film, prepared by gelation/crystallization from solutions, were studied by the effect of nickel particles on the carbonization at various temperatures under argon atmosphere. The carbonized PAN at $800{ }^{\circ} \mathrm{C}$ showed thread-like structures extended from nickel particles. At $1000{ }^{\circ} \mathrm{C}$, the thread-like structures changed to rod-like structures whose one end side was closed by a nickel particle or the aggregation. The disruption of rod-like structures occurred at $1200^{\circ} \mathrm{C}$, and the carbonization at $1400^{\circ} \mathrm{C}$ provided new growth of carbon layers on the surface of spherulitic nickel particles. At $1600^{\circ} \mathrm{C}$, fibrous textures were observed as residual traces of disrupted carbon layers by the overflow of melted nickel due to the thermal expansion. The graphitization degrees for G- and T-components in the films carbonized at $1600^{\circ} \mathrm{C}$ were investigated on the basis of X-ray diffraction intensity distribution from the (002) plane. The analysis was done in terms of the comparison between the experimental and theoretical diffraction intensity curves. The theoretical calculation was carried out by using a concept concerning the para-crystalline theory proposed by Hoseman and Bagchi from the viewpoint of the lattice fluctuation of the c-axis. [doi:10.1295/polymj.PJ2006194]

KEY WORDS Structural Changes of Polyacrylonitrile / Nickel Particles / Catalytic Effect / Carbonization / Para-Crystalline Theory /
\end{abstract}

Carbon materials with high-performance and functionality have been prepared by carbonization of organic compounds, such as polyimide, mesophase pitch, rayon, et al. ${ }^{1-5}$ Recent studies suggested that fine particles of transition metals coexisting with these compounds provided considerable catalytic effect on acquiring the graphite structure at lower temperatures. ${ }^{6-10}$ Oya et al. ${ }^{11,12}$ studied the catalytic graphitization of carbonaceous materials by doping different amount of an organic-nickel compound into a phenolic resin. They found that four kinds of carbon were obtained under different carbonization conditions by a high resolution electron microscope and X-ray diffraction. The effects of catalytic graphitization were termed as D-effect, T-effect, A-effect and Tn-effect. On the other hand, Hatori et al. ${ }^{13}$ studied the carbonization process, related to the catalytic effect of nickel and structural change during carbonization of polyimide films. They pointed out that nickel particles played an important catalytic effect to improve graphitization of polyimide. Further studies were carried out by Kiselev et al. ${ }^{14}$ and Bin et al. ${ }^{15}$ for the graphitization degree of polyimide film at lower temperature in terms of catalytic role of nickel particles. Bin et al. studied catalytic effect of nickel particles and structural change during carbonization by using the three-layered polyimide film, and they succeeded to prepare tough graphite film (sheet), with the thickness of $c a .150 \mu \mathrm{m}$.

Polyacrylonitrile (PAN) is also a well-known polymer as precursor to prepare carbon materials. ${ }^{16-18}$ Kruk et al. prepared mesoporous carbons with sharp distribution pore size from PAN by using ordered and disordered mesoporous silica templates, and the detailed characteristics were investigated. ${ }^{16}$ Ryoo also studied the similar structures prepared by using sucrose. ${ }^{17}$ Giunta et al. prepared carbon-silica nanocomposites from the pyrolysis of PAN. ${ }^{18}$ The carbonization has been carried out for electro-spun thin PAN fibers to pursue smooth thermal stabilization and carbonization as well as to avoid the occurrence of defects and voids within the fibers by chemical reactions under the thermal stabilization and carbonization. ${ }^{19-22}$ Recent studies reported that the composite of PAN and multi-wall carbon nanotubes (MWNTs) were prepared by gelation/crystallization from PAN solution containing MWNTs. ${ }^{23-25}$ And the resultant PAN films were carbonized at the temperature between 900$1600{ }^{\circ} \mathrm{C}$ under co-existence of MWNTs. ${ }^{23}$ The tensile modulus and electrical conductivity for the resultant carbon materials with cross-section area of about $0.6 \mathrm{~mm}^{2}$ (thickness $0.3 \mathrm{~mm}$ and width $2 \mathrm{~mm}$ ) reached $18 \mathrm{GPa}$ and $10 \mathrm{~S} / \mathrm{cm}$, respectively.

Apart from the above investigation, the present pa-

${ }^{\dagger}$ To whom correspondence should be addressed (Tel/Fax: +81-742-20-3462, E-mail: m-matsuo@cc.nara-wu.ac.jp). 


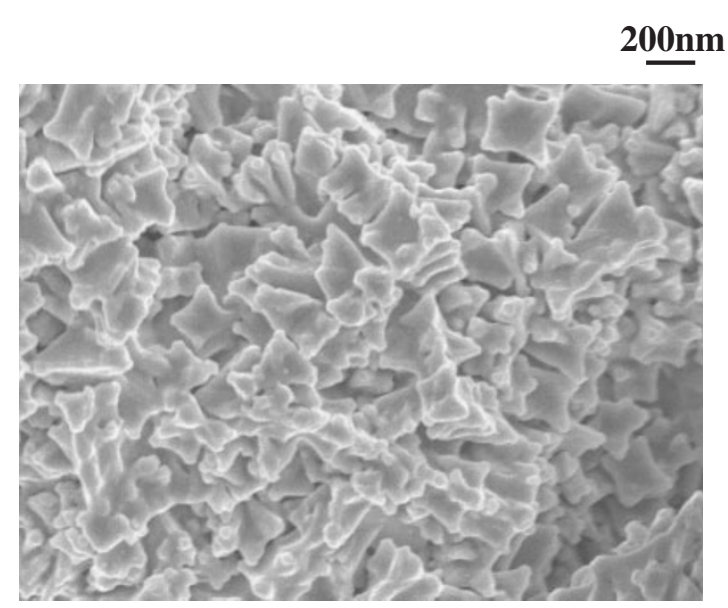

Figure 1. SEM image of the nickel powder.

per deals with the structural change of PAN by the effect of nickel particles during carbonization. The drastic structural change of PAN is observed by scanning electron microscopy in detail after the composite films were carbonized at temperatures beyond $800^{\circ} \mathrm{C}$. The carbonizations at various temperatures provide thread-like, rod-like and spherulitic structures. Of course, such drastic changes have never been confirmed for polyimide ${ }^{15}$ and phenolic resins. ${ }^{12}$ The graphitization degree is analyzed for films carbonized at $1600^{\circ} \mathrm{C}$ by $\mathrm{X}$-ray diffraction techniques. The quantitative analysis for the G- and T-components are also pursued by using a concept for the para-crystalline theory proposed by Hoseman and Bagchi $^{26}$ from a viewpoint of the fluctuation of lattice distance between the (002) planes of the graphite crystal unit.

\section{EXPERIMENTAL}

\section{Sample Preparation}

PAN homopolymer powder with an average molecular weight $\left(M_{\mathrm{w}}=5 \times 10^{5}\right)$ was furnished by the Mitsubishi Rayon Co. Ltd. Figure 1 shows SEM image of nickel particles with diameter of 100$200 \mathrm{~nm}$, purchased from Mitsuwa's Pure Chemical Co. Ltd.

In the present study, the gelation/crystallization technique was used to prepare PAN films and the composite films of PAN and nickel particles. The solvent was dimethylsulfoxide (DMSO). The solution containing $6 \mathrm{~g} / 100 \mathrm{~mL}$ of PAN was prepared by heating the well-blended polymer/solvent mixture at $110^{\circ} \mathrm{C}$ for $30 \mathrm{~min}$ under nitrogen. The solution was quenched by pouring it into a Petri dish, and the dish was put into a refrigerator at $-10^{\circ} \mathrm{C}$ for $c a$. $2 \mathrm{~h}$ immediately, thus generating a gel. After then, the PAN layer was dried at cabinet dryer for $48 \mathrm{~h}$. As the next step,

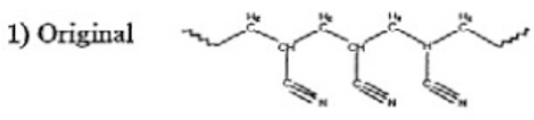

2) Stabilized

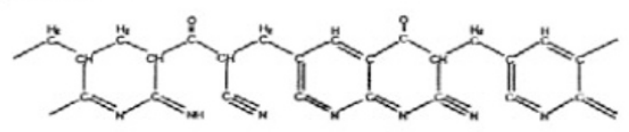

3) Carbonized

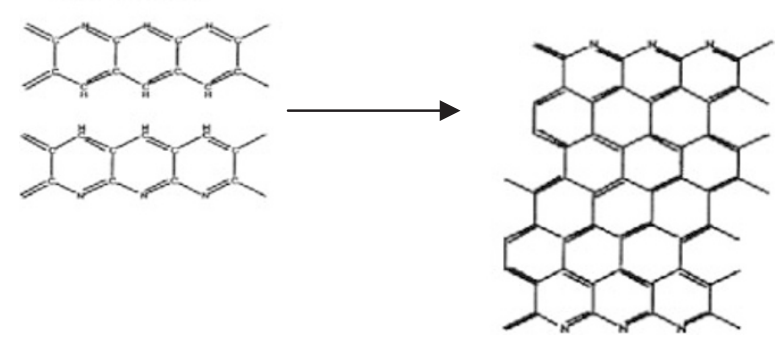

Figure 2. Chemical changes for PAN precursor during different heat-treatment stages.

the PAN solution containing the nickel particles was prepared at the same stirring condition, and the solution was put on the first layer of half-dried PAN film. Two kinds of specimen were prepared, in which the contents of nickel particles against $1 \mathrm{~g}$ PAN were 13.6 and $6.8 \mathrm{mmol}(0.8$ and $0.4 \mathrm{~g})$. The gelation of the PAN solution containing the nickel particles was done by the same method as the preparation of the first layer. After $48 \mathrm{~h}$, PVA solution prepared as the first step was put on the above two layers. The three-layered gel films, of which layers can not be identified by eye observation, were put at cabinet dryer for few days to remove the solvent, and then vacuumdried for few days to remove the residual trace of solvent as much as possible. The thickness of each film is $c a$. $50 \mu \mathrm{m}$, and the thickness of the resultant three-layered film becomes $c a$. $150 \mu \mathrm{m}$.

\section{Stabilization and Carbonization of Sample}

The thermal stabilization of original three-layered film was carried out under the oxidizing atmosphere. The heating rate was $4{ }^{\circ} \mathrm{C} / \mathrm{min}$ to $120^{\circ} \mathrm{C}$, and beyond $120^{\circ} \mathrm{C}$ it was switched to $1{ }^{\circ} \mathrm{C} / \mathrm{min}$ to $270^{\circ} \mathrm{C}$. The heated film was maintained for $60 \mathrm{~min}$. In this treatment, thermoplastic PAN was converted to non-plastic cyclic or ladder compound. ${ }^{14}$ The stabilized films were then treated in argon gas to promote carbonization. The heating rate from room temperature to $500^{\circ} \mathrm{C}$ was $4{ }^{\circ} \mathrm{C} / \mathrm{min}$, and from 500 to $1600^{\circ} \mathrm{C}$ the rate was $8{ }^{\circ} \mathrm{C} / \mathrm{min}$. The heated specimens were maintained at $1600^{\circ} \mathrm{C}$ for desired periods, $0.5,2$ and $5 \mathrm{~h}$, and then cooled down to room temperature naturally. The chemical reactions at various stages of thermal stabilization and carbonization are well-known diagrams, which are shown in Figure 2. 

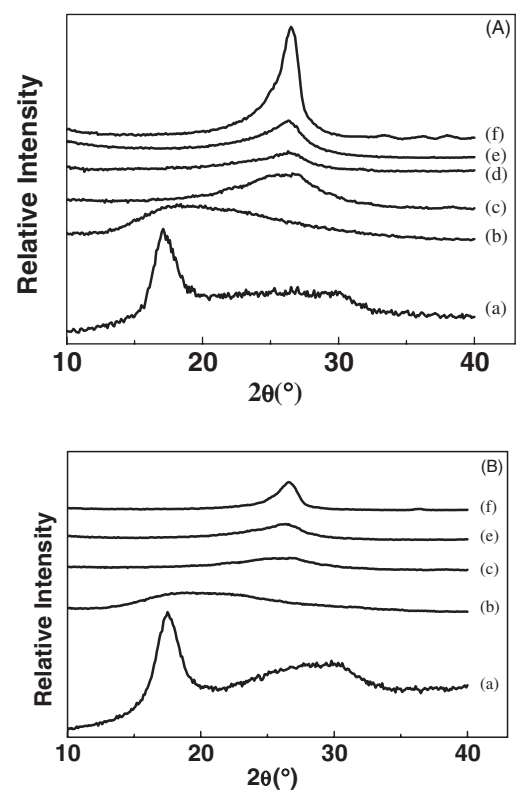

Figure 3. X-Ray diffraction intensity distribution for the specimens heat-treated at different temperatures: (A) $13.6 \mathrm{mmol}$ and (B) $6.8 \mathrm{mmol} \mathrm{Ni}$ particles (Curves: (a) $25^{\circ} \mathrm{C}$, (b) $270^{\circ} \mathrm{C}$, (c) $800^{\circ} \mathrm{C}$, (d) $1000^{\circ} \mathrm{C}$, (e) $1200^{\circ} \mathrm{C}$ and (f) $1600^{\circ} \mathrm{C}$ ).

\section{Analysis Method}

Scanning electron micrographs (SEM) with energy dispersion X-ray spectrometry (EDS) (JEOL 6700) were used to observe the cross-section.

X-Ray diffraction intensity distributions were measured by a $12 \mathrm{~kW}$ rotating-anode X-ray generator (Rigaku RAD-rA) with the monochromatic $\mathrm{CuK} \alpha$ radiation (wavelength of $0.1542 \mathrm{~nm}$ ). The measurement by a reflection method was done from $5^{\circ}$ to $40^{\circ}$ (twice the Bragg angle) with a step-scanning device at a step interval of $0.05^{\circ}$, each fixed time of $100 \mathrm{~s}$. Before these measurements, X-ray diffraction patterns (end view) were observed for the specimens carbonized at $800,1000,1200$ and $1600^{\circ} \mathrm{C}$, when an incident $\mathrm{X}$-ray beam was directed along the film surface direction. The random orientation of turbostratic structures was confirmed for the all carbonized specimens.

Thermo-gravimetric analysis (TGA) measurement was done using TG/DTA 6300 (SII Nano Technology Inc.); the specimens was heated under nitrogen from room temperature to $1500^{\circ} \mathrm{C}$ at a heating rate of $10^{\circ} \mathrm{C} / \mathrm{min}$. Electrical conductivity at room temperature was measured by high resistance measuring device (HR-100) produced by Iwamoto Seisakusho Co. Ltd.

\section{RESULTS AND DISCUSSION}

Figure 3(A) and (B) show X-ray diffraction intensity for the composite film carbonized for $30 \mathrm{~min}$ in the presence of nickel particles of 13.6 and $6.8 \mathrm{mmol}$, re-

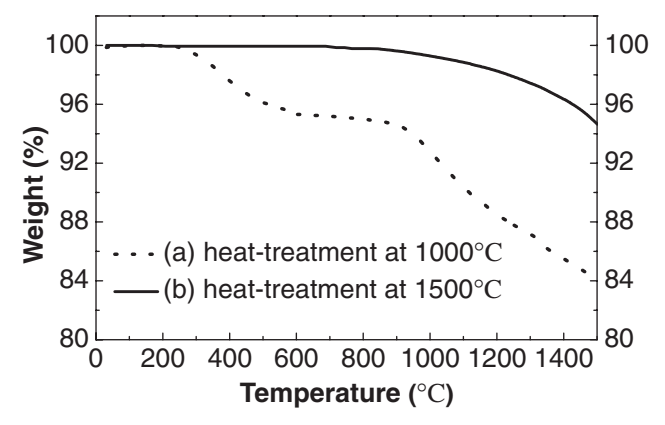

Figure 4. TG curves of the specimens carbonized at 1000 and $1600{ }^{\circ} \mathrm{C}$ in the presence of $13.6 \mathrm{mmol}$ nickel particles.

spectively, in the range of twice the Bragg angles (2 $\theta$ ) from 10 to $40^{\circ}$. The range was fixed to avoid the appearance of strong peaks from nickel crystallites. The two diffraction peaks from orthorhombic PAN can be observed at $2 \theta_{\mathrm{B}}=17.0$ and $29.5^{\circ}$, respectively, but the peaks disappeared for the composite film stabilized at $270^{\circ} \mathrm{C}$, indicating complete disruption of the PAN crystallites by the chemical reactions associated with oxidation, dehydrogenation, and cyclization. At $800^{\circ} \mathrm{C}$, a very broad peak from the $(002)$ plane appeared at $c a .26^{\circ}$, and the peak intensity increases slightly as the carbonization temperature increases, confirming that stabilized PAN crystallities changed to turbostratic organization of the carbon layer plane. Detailed observation reveals that the diffraction peak from the (002) plane of the carbonized PAN film shifts to higher diffraction angle as the heating temperature increases, indicating that the average distance between the graphene sheets became narrower. At the same heat-treated temperature, the peak magnitude for the composite film with $13.6 \mathrm{mmol}$ nickel particles is more intense than that with $6.8 \mathrm{mmol}$. Furthermore, the diffraction peak for the specimen carbonized in the absence of nickel particle $(0 \mathrm{mmol})$ was confirmed to be very weak. The diffraction curves in Figure 3 indicated that the growth of turbostratic (quasi graphite) structure is more pronounced with increasing the content of nickel particles, and the nickel particles play an important catalytic effect to improve the carbonization of PAN. Accordingly, the following discussions are mainly done for the specimen containing $13.6 \mathrm{mmol}$ nickel particles.

Thermal stability of the carbonized specimens was investigated by TGA measurement to check the carbonization degree estimated by the X-ray diffraction. Figure 4 shows the results, in which curves (a) and (b) show the weight loss for the specimens carbonized at 1000 and $1600^{\circ} \mathrm{C}$, respectively. The TGA measurements were done from $30^{\circ} \mathrm{C}$ to $1500^{\circ} \mathrm{C}$. It is seen that curve (a) has two beginning steps of distinct weight loss at $250^{\circ} \mathrm{C}$ and $900^{\circ} \mathrm{C}$. The first step might be due to pyrolysis of the stabilized PAN (not carbon- 

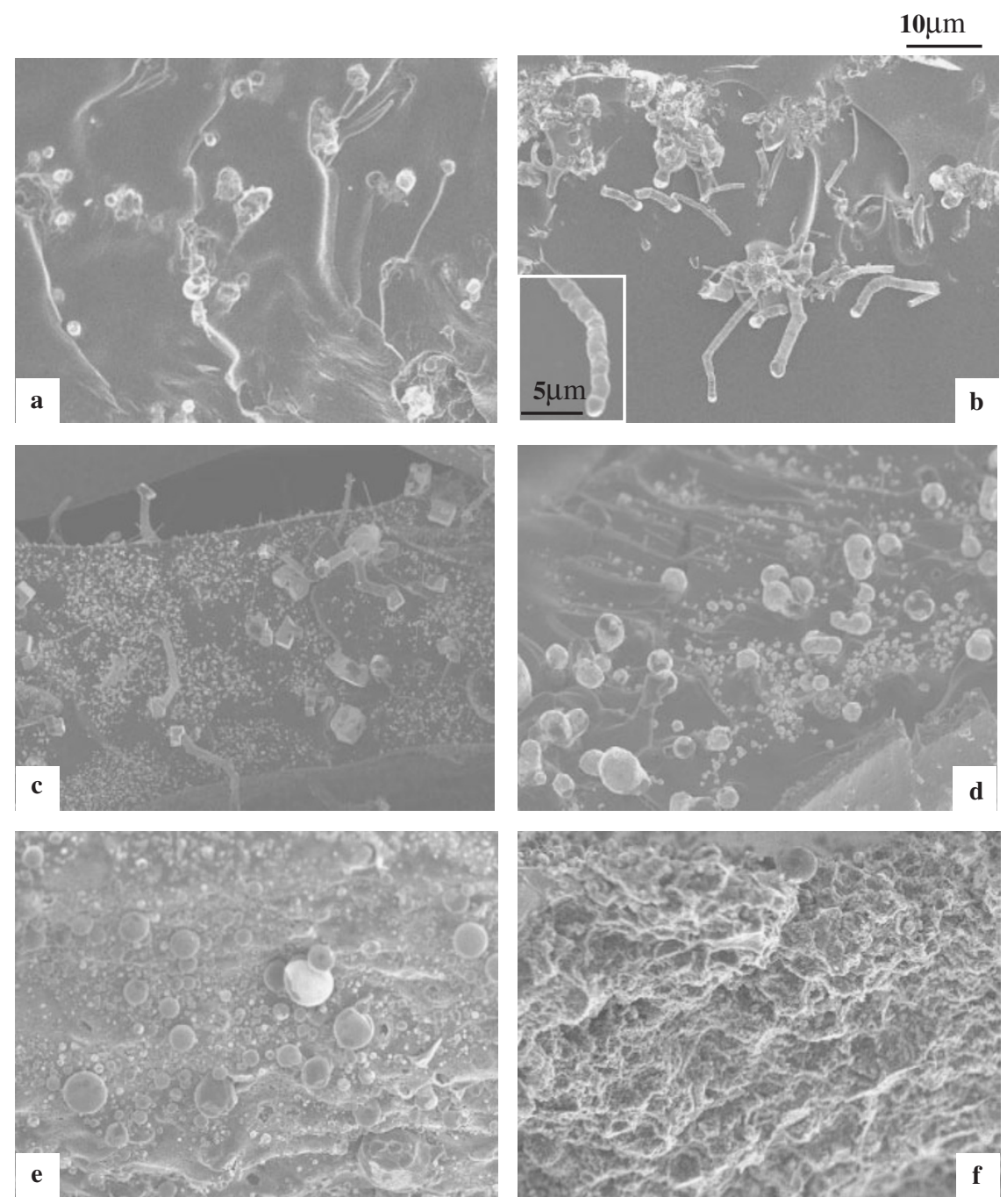

Figure 5. SEM images of the cross-section of specimens carbonized at different temperatures: (a) $800^{\circ} \mathrm{C}$, (b) $1000^{\circ} \mathrm{C}$, (c) $1200{ }^{\circ} \mathrm{C}$, (d) $1300^{\circ} \mathrm{C}$, (e) $1400^{\circ} \mathrm{C}$ and (f) $1600^{\circ} \mathrm{C}$.

ized). With the increase in temperature, the amorphous carbon was partly responsible for the second weight loss. This weight loss was mainly caused by the attacked of impurity of turbostratic structure containing $\mathrm{O}_{2}, \mathrm{CO}_{2}$ and $\mathrm{H}_{2} \mathrm{O}$, and such thermal behavior indicates poor carbonization of the specimen heattreated at $1000{ }^{\circ} \mathrm{C}$. On the other hand, as shown in curve (b), the weight loss of the specimen carbonized at $1600^{\circ} \mathrm{C}$ started over $800^{\circ} \mathrm{C}$, but the total weight loss up to $1500^{\circ} \mathrm{C}$ was only $5 \%$, indicating pure carbon turbostratic structure.

Figure 5 shows SEM photographs of the cross-section of the specimens, nickel content being $13.6 \mathrm{mmol}$ against $1 \mathrm{~g}$ PAN, carbonized at the indicated temperatures for $30 \mathrm{~min}$. The images at each carbonized temperatures were confirmed for the four specimens. Image (a) shows thin extended thread-like structures with impurity turbostratic structures produced by chemical reaction of PAN under the heat-treatment at $800^{\circ} \mathrm{C}$. The growth of the thread-like structures started from the surface of nickel particles. Such structural growth justifies the catalytic effect of nickel particles on the carbonization. Of course, no appearance of carbonized structure and no X-ray diffraction peak were confirmed for the specimen carbonized in the absence of nickel particles. Judging from a broad peak of the X-ray intensity curve (c) in Figure 3(A), the thread structure composed of stacking formation of the cyclization segments with a lattice distance close to the space between the (002) planes of the graphite crystallites, but it is evident that the lattice distance has large fluctuation. The stacking formation is probably associated with the structure usually known as the initial ladder structure. As shown in image (b), carbonization at $1000^{\circ} \mathrm{C}$ provided rod-like structures with the regular knots along the longitudinal axis, but the length and width of each structure are different. Interestingly, the one end side of each rod is closed by a nickel particle or the aggregation shown as a white spherulite, which can be evidenced 
from EDS results. The existence of nickel particles on one end side suggests that the rod-like structures are attributed to the growth of the thread-like structures. Here we must emphasize that the structural growth extended from the nickel particles in (a) and (b) is characteristics of carbonization of PAN, and such structures have never been observed under the carbonization of polyimide film containing nickel particles.

As shown image (c), most of rod-like structures were disrupted at $1200^{\circ} \mathrm{C}$, and the resultant cubic particles were dispersed. In addition to the cubic particles, there are a number of small structures whose average size is about $100 \mathrm{~nm}$ indicating nickel particles. At $1300^{\circ} \mathrm{C}$, the cubic structures tend to change spherulitic structures as shown in image (d), and this tendency becomes more considerable by the carbonization at $1400^{\circ} \mathrm{C}$ as shown in image (e). Judging from the carbonization of polyimide and nickel composites reported by Lamber et al. ${ }^{27}$ and Bin et al.,${ }^{15}$ it may be expected that the carbonization occurs on the surface of nickel supherulites, and most of particles are surrounded by carbon layers with increasing temperature. Image (f) shows broken carbonaceous structure just as fibrous like textures of the specimen carbonized at $1600^{\circ} \mathrm{C}$, in which most of circular particles disappeared. To explain the appearance of the fibrous textures, the enlarged photograph concerning the carbonization at $1400^{\circ} \mathrm{C}$ is shown in Figures 6 and 7. Of course, the carbonization thermal behavior was confirmed for the specimen whose nickel content against $1 \mathrm{~g}$ PAN was $6.8 \mathrm{mmol}$. Here it should be noted that such drastic changes observed for PAN have never been observed for polyimide ${ }^{15}$ and phenolic resins ${ }^{12}$ but the reason remains resolved problems.

Figure 6 shows an EDS photo obtained by the LEI (lower secondary electron image) method and element spectrum for a circular particles in the specimen, nickel content being $13.6 \mathrm{mmol}$ against $1 \mathrm{~g}$ PAN, heated up at $1400^{\circ} \mathrm{C}$. As postulated from established papers, the large nickel spherulites are thought to be the aggregation of melt nickel particles in the presence of carbon and energy irradiation was done for a large spherulite to obtain element spectra from the selected points on the particle surface. The surface of the large particle shows dark and white parts assigned as 001 and 002, respectively. LEI, being different from the SEI (secondary electron image), could observe the deep part of the sample surface. Element spectra indicated that white part is surrounded by thin carbon layer, while the dark part of the surface was covered by thick carbon layer because of the equal intensity ratio of carbon against nickel (1:1). This means that the carbonization started on the surface of nickel spherulites and most of spherulites are surrounded by carbon layers with progressing carbonization, just as pointed out
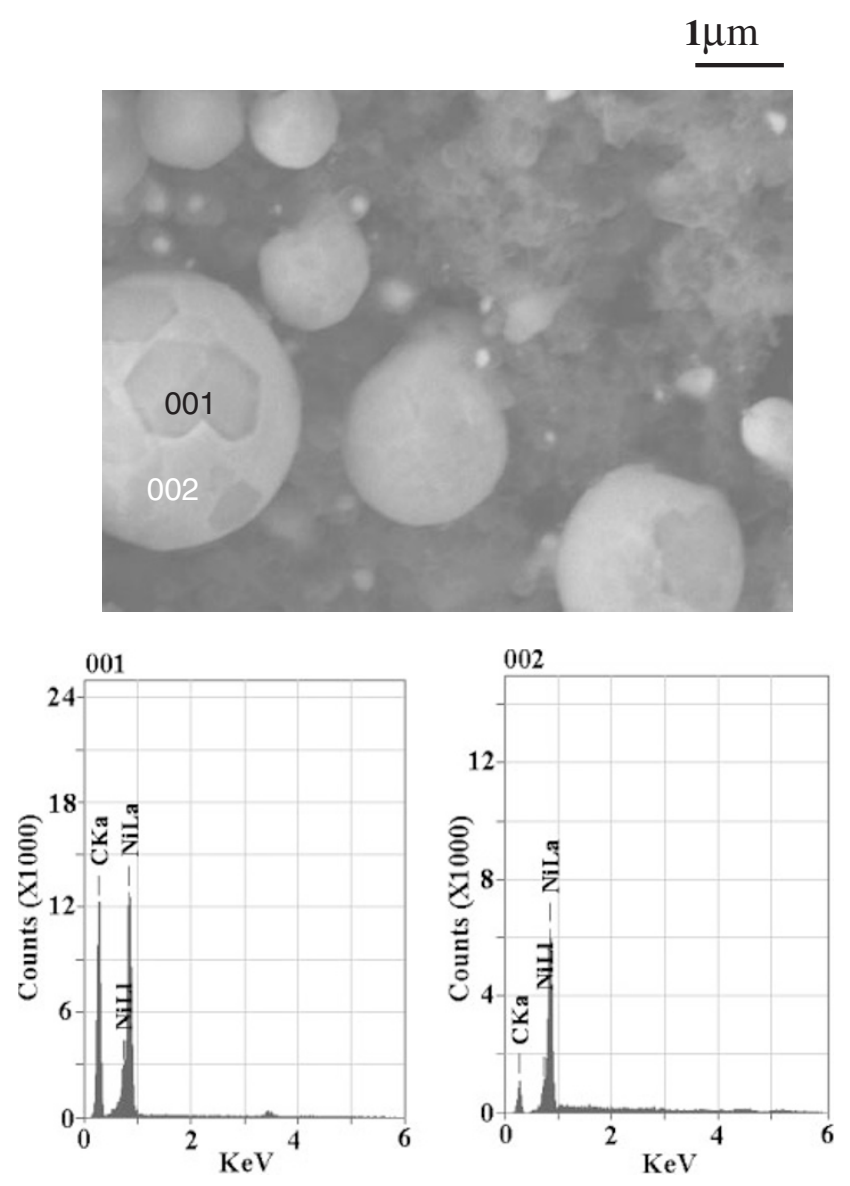

Figure 6. EDS dot analysis of specimens carbonized at $1400^{\circ} \mathrm{C}$.

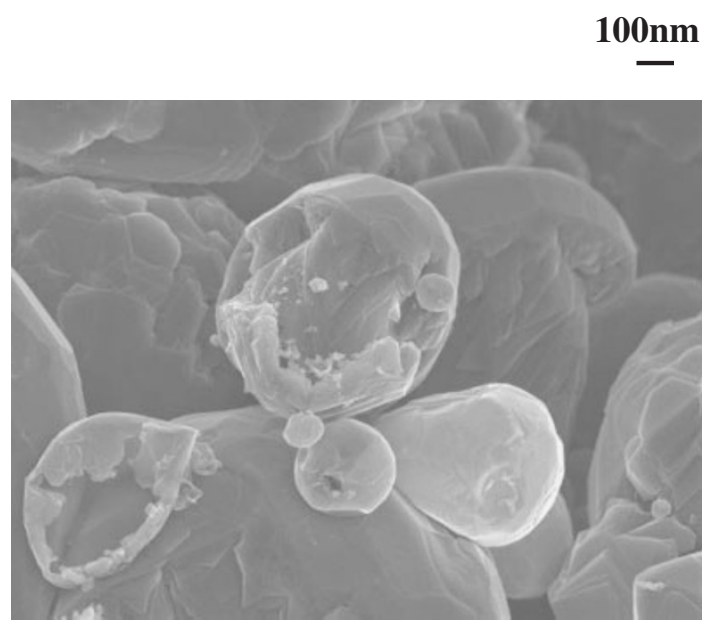

Figure 7. SEM image observed for the disruption of the spherulitic structures in specimen carbonized at $1400^{\circ} \mathrm{C}$.

for polyimide and nickel composite by Lamber et al. ${ }^{27}$

Figure 7 shows the disruption of the carbon layers for the specimen carbonized at $1400^{\circ} \mathrm{C}$. It is evident that the nickel particles were covered by carbon layer, but the outflow of the melted nickel probably occurred through the disruption of carbon layers due to the vol- 

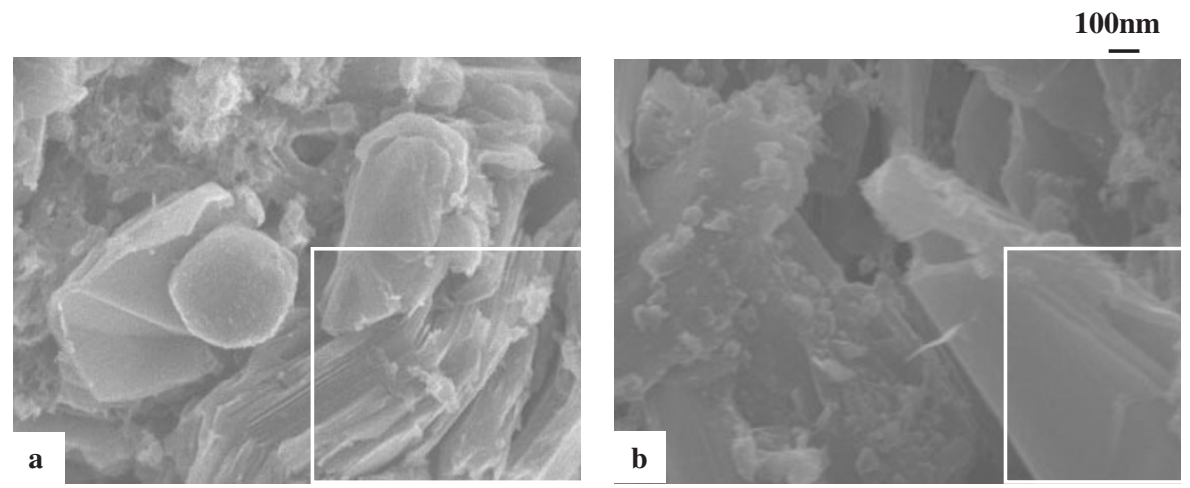

Figure 8. SEM images of the stacking structure of specimen carbonized at (a) $1400^{\circ} \mathrm{C}$ and (b) $1600{ }^{\circ} \mathrm{C}$.

ume expansion of nickel particles.

Here we must emphasize that the clear stacking structure of graphite layers can be observed locally in the specimens heated up to 1400 and $1600^{\circ} \mathrm{C}$, which are shown in the white frames in Figure 8(a) and (b). Obviously, the layers are in less ordered structure in comparison with the polyimide-nickel composite carbonized at $2800{ }^{\circ} \mathrm{C} .{ }^{13}$ It was also confirmed that Raman spectrum gives high peak of D-band and small peak of G-band, indicating that most of crystallites within the carbonized composite contain turbostatic (quasi-graphite) structures with the fluctuation of the lattice distance within the crystallites.

To solve the above question, detailed analysis of the X-ray diffraction must be done in comparison between the experimental and theoretical diffraction intensity curves. In doing so, first of all, experimental diffraction curves must be obtained by the correction for air scattering, polarization and absorption.

Figure 9(a) and (b) show the corrected X-ray diffraction intensity (represented as circles) for the films carbonized for 0.5 and $2 \mathrm{~h}$, respectively, at $1600^{\circ} \mathrm{C}$. The profile for the specimen carbonized for $5 \mathrm{~h}$ was similar to the two experimental curve profiles, indicating the independence of the holding period. The experimental intensity was classified into three components by assuming three kinds of Gauss function. The intensity curves from the respective crystal and amorphous components are shown as dotted curves, and the total intensity curves from these composites are shown as solid curves. The total intensity curves are in good agreement with the experimental intensity plots. The higher and lower peaks appeared at $c a$. 26.5 and $26.0^{\circ}$, respectively. It is expected that the higher peak appeared at $c a .26 .5^{\circ}$ is due to the diffraction from turbostratic structure with small lattice fluctuation termed as G-component by Otani et al. ${ }^{28}$ while the lower peak at $c a \cdot 26.0^{\circ}$, from the turbostratic structure with large fluctuation termed as T-component. The peak at $c a \cdot 26.0^{\circ}$ did not appear usually
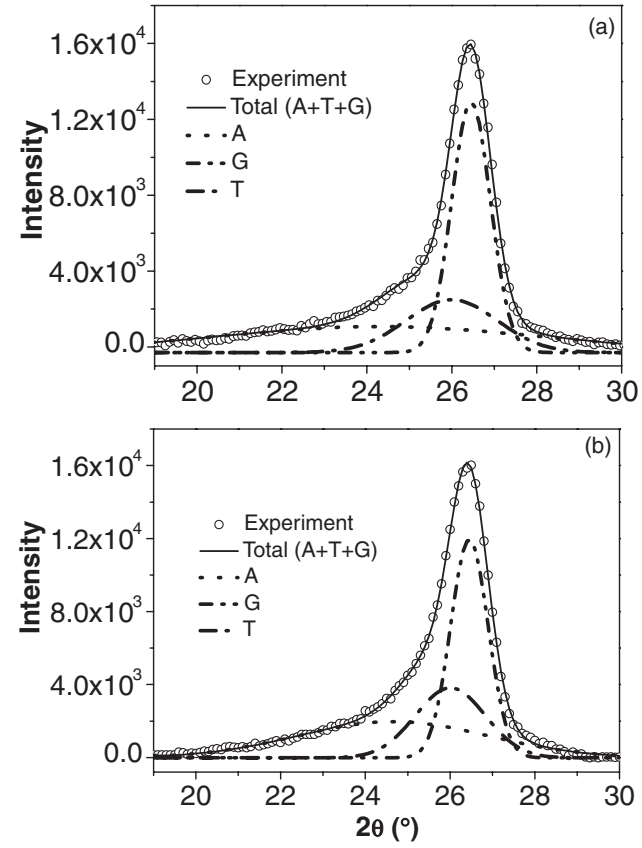

Figure 9. The corrected $\mathrm{X}$-ray diffraction curves for the specimens carbonized at $1600^{\circ} \mathrm{C}$ for a time period of (a) $0.5 \mathrm{~h}$ and (b) $2 \mathrm{~h}$.

for carbon films derived from polyimide containing nickel particles. Then it is suggested that the degree of graphitization of turbostratic structures from PAN precursor at $1600{ }^{\circ} \mathrm{C}$ is much less than that from polyimide prepared at the same condition. ${ }^{15}$ The average ratio of G-component to total-component estimated for four specimens carbonized at $1600^{\circ} \mathrm{C}$ was $c a$. 0.75 .

Otani et $a .^{28}$ reported that carbons derived from phenolic resin by catalytic action of nickel particles depended on the nickel contents. Namely, the peak at $c a .26^{\circ}$ became more intense with decreasing nickel content. The peak position shifts to higher degree with increasing nickel content and carbonization temperature, and the diffraction intensity from carbon heattreated at $1600{ }^{\circ} \mathrm{C}$ showed overlapped curves with 

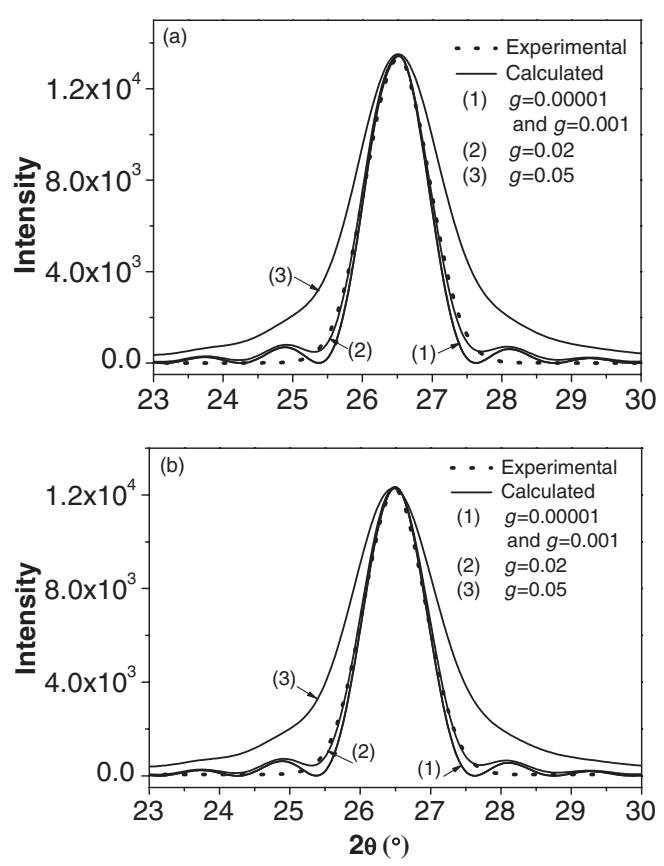

Figure 10. The real peak curves for the G-component of specimen heat-treated for (a) $0.5 \mathrm{~h}$ and (b) $2 \mathrm{~h}$.
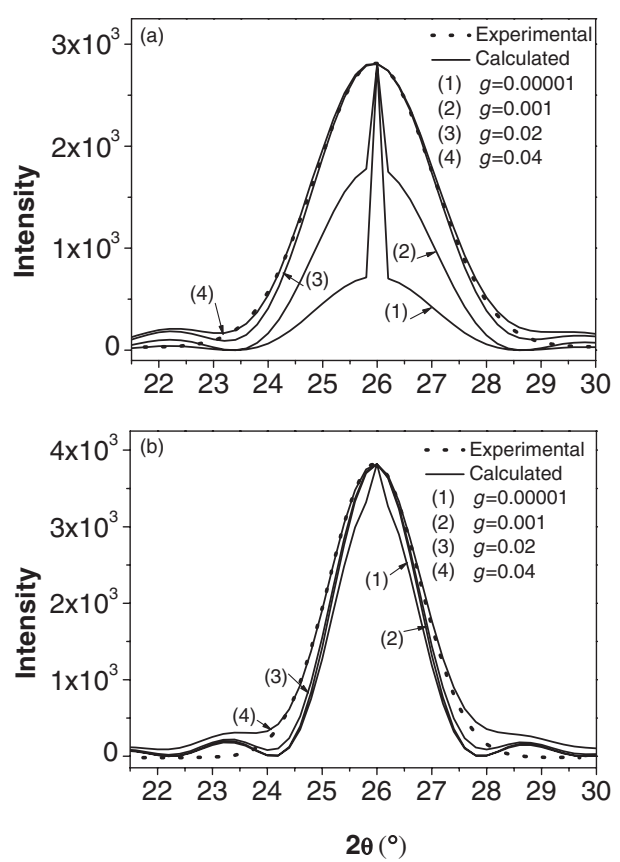

Figure 11. The real peak curves for the T-component of specimen heat-treated for (a) $0.5 \mathrm{~h}$ and (b) $2 \mathrm{~h}$.

Table I. Crystal size of graphite or turbostatic crystallites of the film carbonized at indicated condition

\begin{tabular}{lllll}
\hline & $\begin{array}{l}\text { Heat-treated } 0.5 \mathrm{~h} \\
\text { T-component }\end{array}$ & G-component & $\begin{array}{l}\text { Heat-treated } 2 \mathrm{~h} \\
\text { T-component }\end{array}$ & G-component \\
\hline $1000^{\circ} \mathrm{C}$ & $3.57 \mathrm{~nm}$ & $3.74 \mathrm{~nm}$ & - & - \\
& $2 \theta=25.999^{\circ}$ & $2 \theta=26.450^{\circ}$ & - & $8.07 \mathrm{~nm}$ \\
$1600^{\circ} \mathrm{C}$ & $4.24 \mathrm{~nm}$ & $7.91 \mathrm{~nm}$ & $4.80 \mathrm{~nm}$ & $2 \theta=26.502^{\circ}$ \\
& $2 \theta=25.999^{\circ}$ & $2 \theta=26.502^{\circ}$ & $2 \theta=25.999^{\circ}$ & $2 \theta$ \\
\hline
\end{tabular}

peak tops at $c a .26 .5$ and $26.0^{\circ}$. Their results support the present experimental results.

The dotted curves in Figure 10(a) and (b) reveal the real peak profile for the G-component in the specimens annealed for 0.5 and $2 \mathrm{~h}$, respectively, and those in Figure 11(a) and (b) reveal the profiles of the corresponding T-component. The experimental dotted curves were obtained by using convolution of two intensity distributions obtained for quartz particles, and each test specimen in order to analyze the lattice fluctuation of the G- and T-components. This method was described elsewhere. ${ }^{15}$ Table I shows approximate crystallite sizes of different kinds of carbons within the films carbonized for the indicated period at $1600^{\circ} \mathrm{C}$. The approximate crystal sizes were calculated roughly by Scherrer's equation, in which the constant value of $\mathrm{K}$ is 0.9 . As listed in Table I, the average size along the c-axis of the G-component is $c a$. $8.07 \mathrm{~nm}$ containing twelve crystal unit, and average half length $(\bar{c} / 2)$ of $\mathrm{c}$-axis corresponding to the distance between the $(002)$ planes is $0.6727 \mathrm{~nm}$. The average size of the T-component is $4.80 \mathrm{~nm}$ contain- ing seven crystal unit, and $(\bar{c} / 2)$ is $0.6855 \mathrm{~nm}$. The obtained values contain experimental error because of the film thickness of $c a .150 \mu \mathrm{m}$. Because, the diffraction peak is obliged to shift to higher or lower value of the twice Bragg angle with increasing the film thickness, since the number of the crystal planes deviated from the real reflection point of the optical system increases. ${ }^{15,29,30}$ The crystal sizes were smaller than that in the carbon films derived from polyimide and nickel composites by heat-treatment at $1600^{\circ} \mathrm{C}$, and also much smaller than the reported value $(300 \mathrm{~nm})$ for natural graphite crystal. ${ }^{31}$

Here it should be noted that Scherrer's equation cannot be essentially applied to imperfect crystallites but it was used to estimate the size of imperfect crystallites approximately. Because the number of unit cell along the c-axis is needed as a parameter to pursue the theoretical calculation. On the basis of the number estimated by Scherrer's equation as listed in Table I, the accurate sizes of the imperfect crystallites in the present paper were estimated by curve fitting between the experimental and theoretical curves by 
considering the lattice fluctuation along the c-axis, as discussed below.

According to the X-ray diffraction theory, the diffraction intensity from perfect crystal units is given by $I=F(h, k, \ell) F^{*}(h, k, \ell) \frac{\sin ^{2} \pi h N_{a}}{\sin ^{2} \pi h} \frac{\sin ^{2} \pi k N_{b}}{\sin ^{2} \pi k} \frac{\sin ^{2} \pi \ell N_{c}}{\sin ^{2} \pi \ell}$

where

$$
F(h, k, \ell)=\sum f_{j} \exp \left\{2 \pi i\left(h x_{j}+k y_{j}+\ell z_{j}\right)\right\}
$$

In eq $1, F^{*}(h, k, \ell)$ is the complex conjugate of $F(h, k, \ell)$ and $N_{a}, N_{b}$, and $N_{c}$ are the number of crystal unit cell along the a-, b- and c-axes, respectively and $h, k$ and $\ell$ are the Miller indexes. The atom structure factor of $f_{j}$ is for the carbon atom in a graphite crystal unit.

Apart from eq 1, the present theory is focused on the profile of diffraction peak from the (002) plane to study the degree of fluctuation of crystal lattice along the c-axis. In this case, the numbers of the unit cell along the a-axis as well as that along the b-axis are dependent upon the magnitude of the diffraction intensity, but independent of the peak profile. The following calculation is carried out only for the fluctuation of the lattice distance along the c-axis on the two-dimensional plane, in which $\mathrm{X}_{1}$ and $\mathrm{X}_{2}$ axes are in the directions of the film thickness and film surface, respectively.

Model (a) in Figure 12 shows the two-dimensional schematic diagram of lattice arrangement in the direction of the c-axis of graphite crystal, and the model (b) shows the crystal unit cell. In model (a), $\mathbf{c}_{j}$ is a displacement vector denoting the length of the c-axis of the j-th unit cell. $\mathbf{R}_{j}$ denotes a vector from the center (O) to a carbon atom within the j-th unit cell represented as a solid circle. Judging from that the atom structure factor $f_{j}$ is only for carbon.

The function $H(c)$ for the possibility of finding the nearest-neighbor chain at a distance of the c-

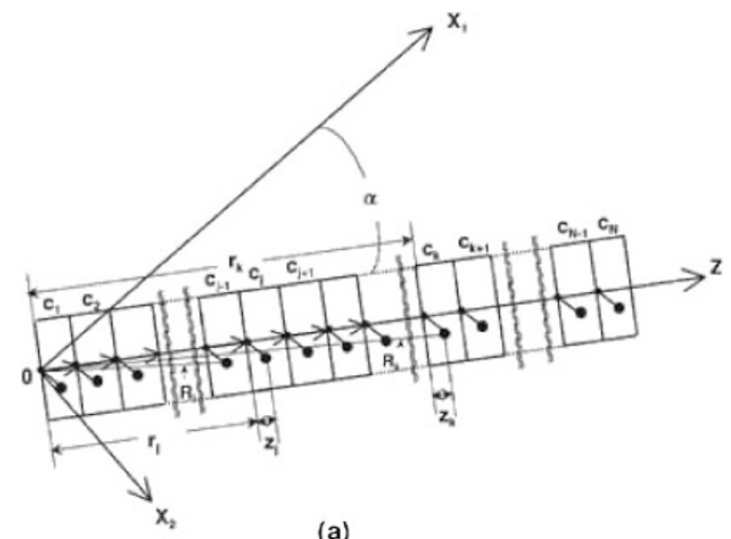

(a)

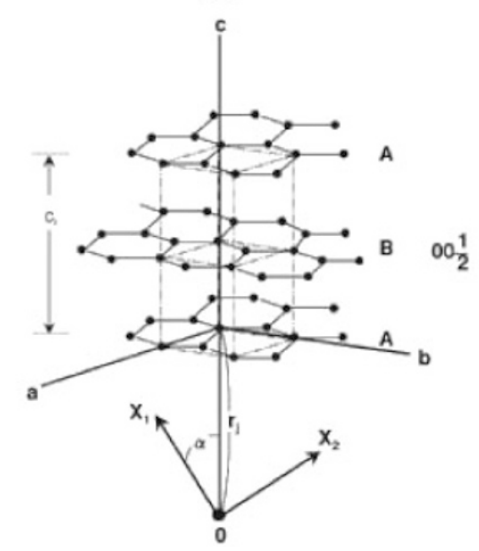

(b)

Figure 12. Model (a) shows the schematic diagram of lattice arrangement in the direction of the c-axis of graphite crystal and the model (b), the crystal unit cell.

axis is represented by the function by Hoseman and Bagchi. ${ }^{26}$ In this model system, $H(c)$ may be given by

$$
H(c)=\frac{1}{\sqrt{2 \pi \Delta c^{2}}} \exp \left\{-\frac{(c-\bar{c})}{2 \Delta c^{2}}\right\}
$$

where $\bar{c}$ is the average length of the c-axis and $\Delta c^{2}$ is the mean square fluctuations of the displacement of the c-axis.

Following the previous mathematical treatments, ${ }^{15}$ the normalized diffraction intensity is given by

$$
I(\theta)=8 f_{c}^{2}\left[1-\frac{1}{2}\left(\frac{\Delta c}{\bar{c}}\right)^{2}\left\{\frac{2 \pi \bar{c}}{\lambda} \sin \theta \cos (\alpha-\mu)\right\}^{2}\right]\left\{Z(\theta)-\frac{I_{C}(\theta)}{N_{3}}\right\}
$$

where $\theta$ and $\mu$ are diffraction and azimuthal angles, respectively, of the diffraction beam, and $\alpha$ is the polar angle between $\mathrm{X}_{1}$ and $z(c)$ axes. To calculate the curve profile of the (002) plane, $N_{3}$ must be set to be the twice of the number of crystal unit $\left(N_{\mathrm{c}}\right)$ along the c-axis. In eq 4 , the inter-chain interference effect from the chains becomes stronger with decreasing the disordered parameter $g=(\Delta c / \bar{c})$, while the effect becomes neglected when $\mathrm{g}$ is infinite. In eq $4, Z(\theta)$ and $I_{C}(\theta)$ are described elsewhere. ${ }^{15}$

Here it is of reasonable to consider that the number of graphite crystal units has distribution. Since the crystallite size of graphite is much smaller than the irradiated area by X-ray beam, there exist graphite crystallites with several sizes within the irradiated area. ${ }^{32-34}$ In the present paper, the distribution for the number of crystal units is given by a Gaussian distribution as follows: 


$$
P\left(N_{3}\right)=\frac{\exp \left\{-\frac{\left(N_{3}-\bar{N}_{3}\right)}{2 \sigma_{N_{3}}{ }^{2}}\right\}}{\sum_{N_{3}=1}^{2 \bar{N}_{3}-1} \exp \left\{-\frac{\left(N_{3}-\bar{N}_{3}\right)}{2 \sigma_{N_{3}}{ }^{2}}\right\}}
$$

where $\bar{N}_{3}$ and $\sigma_{N_{3}}$ are the average twice number of crystal units and its standard deviation. Then the average diffraction intensity may be given by

$$
\langle I(\theta)\rangle=\sum_{N_{3}=1}^{2 \bar{N}_{3}-1} P\left(N_{3}\right) \frac{I(\theta)}{N_{3}^{2}}
$$

To give the same peak top between experimental and calculated curves, eq 6 is modified as follows:

$$
I_{\text {cal }}(\theta)=\frac{\langle I(\theta)\rangle}{\left\langle I(\theta)_{\text {top }}\right\rangle} I_{\text {ept }}
$$

where $\left\langle I(\theta)_{\text {top }}\right\rangle$ is the peak top intensity of the calculated intensity $\langle I(\theta)\rangle$, and $\mathrm{I}_{\exp }$ is the peak top intensity of the corrected experimental intensity. Of course, $\langle I(\theta)\rangle /\left\langle I(\theta)_{\text {top }}\right\rangle$ becomes unity at twice the Bragg angle giving the peak top of the calculated intensity. In the present system, the crystallites are oriented randomly within the film. Accordingly, the crystallites oriented at $\alpha=90^{\circ}$ can be detected by scanning Xray counter in the horizontal direction $\left(\mu=90^{\circ}\right)$.

The solid curves in Figure 10 show the experimental and calculated curves of the G-component appeared at $c a .26 .5^{\circ}$, while those in Figure 11 correspond to the T-component appeared at $c a .26 .0^{\circ}$. The comparison was carried out for the specimens carbonized at $1600^{\circ} \mathrm{C}$ for 0.5 and $2 \mathrm{~h}$. The calculation at $\sigma_{N_{3}}=3$ was pursued for the G- and T-components by assuming $\bar{N}_{3}=12$ and 6 , respectively. The curve profiles for the two components mean the independence of the holding time. As can be seen in two figures, the peak profile is strongly dependent upon the disordered parameter $g=(\Delta c / \bar{c})$. The $g$-parameters to give the best fit between experimental and calculated intensity curves were 0.02 and 0.04 for the G- and $\mathrm{T}$-components, respectively. This means that the Tcomponents have the larger fluctuation of lattice distance between the (002) planes in comparison with the G-component similar to the unstable structures. Interestingly, the curves calculated at $g<0.001$ for the G-component showed almost the same profiles and also the curve calculated at $g=0.02$ is almost equal to the curves calculated at $g<0.001$. This means that the peak for the G-component appeared at $c a .26 .5^{\circ}$ has very small lattice fluctuation indicating perfect graphite crystal. Namely, the broad profile for the G-component is attributed not to the imperfectness (fluctuation of lattice distance) but to the few number of crystal unit. According to the report for carboniza-
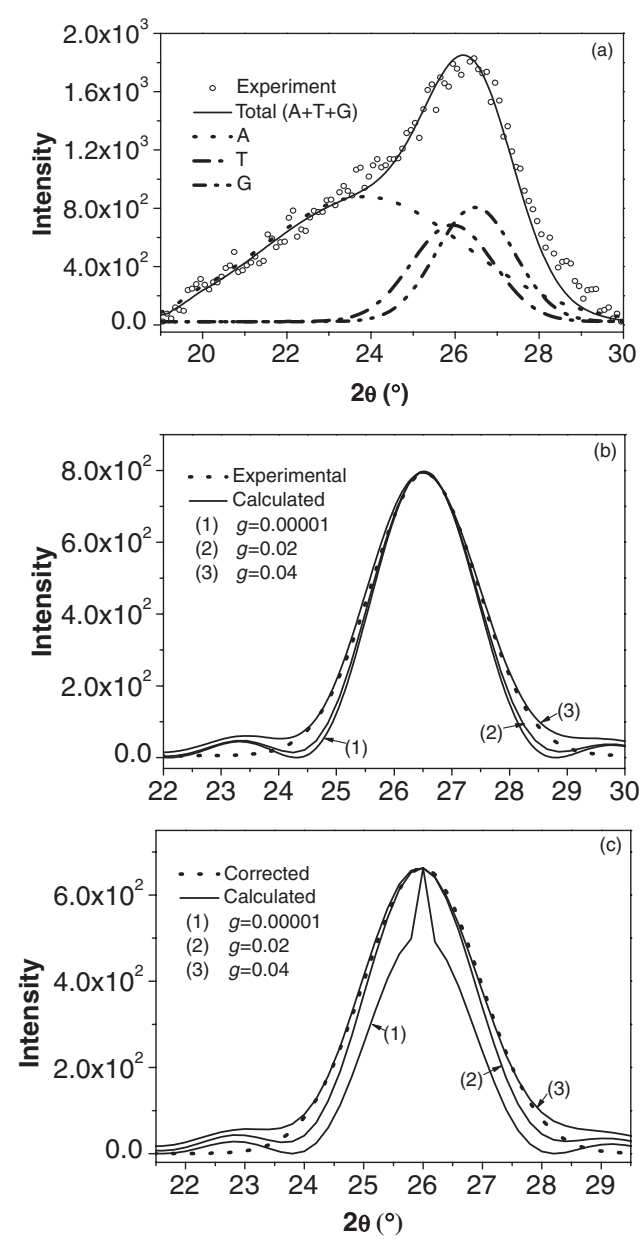

Figure 13. The diffraction intensity distribution for the carbon films heated at $1000^{\circ} \mathrm{C}$ : (a) corrected curve, (b) G-component curve and (c) T-component curve.

tion, the curve profile from polyimide ${ }^{15}$ was much shaper than that of the present curve profile. Such sharpness is attributed to the large size of the carbon crystallites produced from polyimide. In contrast, the peak profiles calculated at $g>0.02$ deviate from the experimental results.

As shown in Figure 11(a) and (b), the curves calculated at $g<0.02$ are poor agreement with the experimental (dotted) curve, while the curves at $g=0.04$ gave the best fit. Furthermore, the calculated curves at $\bar{N}_{3}=12$ were confirmed to provide large derivation from the experimental (dotted) curves in spite of the selection of any $g$ values. This demonstrates that the T-component has small turbostratic structure with the large lattice fluctuation.

Figure 13(a) shows the diffraction intensity distribution for the carbon films carbonized at $1000^{\circ} \mathrm{C}$. The experimental data were plotted as circles, and the experimental results were classified into three components: amorphous, $\mathrm{T}$ - and G-components by assuming three kinds of Gauss function. The corresponding morphology of the carbon shows the rod- 
like structure whose one end side is closed by nickel particles or the aggregation of the particles (see image (b) in Figure 5). As shown in Figure 13(a), the diffraction curve shows the large contribution from amorphous carbon in addition to small T- and G-components. The average ratio of G-component to totalcomponent estimated for four specimens carbonized at $1000^{\circ} \mathrm{C}$ was $c a .0 .55$.

Figure 13(b) and (c) are the comparison between the experimental and calculated curves of the Gand T-components, respectively. The calculations for the G- and T-components were carried out by using $\bar{N}_{3}=6$. The disordered parameter $g$ of the G-component was 0.04 , indicating very small turbostratic structure with very large lattice fluctuation, different from the G-component in the specimen carbonized at $1600^{\circ} \mathrm{C}$. This unstable G-component provided the peak shift to lower diffraction angle (from $26.502^{\circ}$ to $26.450^{\circ}$ ) indicating the expansion from the normal average length of the c-axis, which means crude packing of graphite chains within the crystal unit cell. Furthermore, the $g$-value, 0.04, of the G-component is equal to the value of the T-component. This means that the different characteristic between the G- and T-components in the specimen carbonized at $1000^{\circ} \mathrm{C}$ is only the difference of the average length of the c-axis and the rod-like structures in image (b) shown in Figure 5 composed of unstable G- and T-components in addition to the amorphous carbon region. Anyway, a series of experimental and calculated results provided a conclusion that the average crystal size was very small when PAN as precursor was carbonized under nickel particles as catalyst. This also supports the electric conductivity of the bulk specimen of $c a .66 .9 \mathrm{~S} / \mathrm{cm}$ which is much lower than the conductivity of graphite, because of the existence of amorphous carbon shown in Figure 9 as A-component.

\section{CONCLUSION}

The three-layered PAN film was prepared by gelation/crystallization method. The middle layer composed of nickel particles. The carbonization of the composite film was carried out in the temperature range of $800-1600^{\circ} \mathrm{C}$ in argon atmosphere. The carbonization degree was pronounced with increasing the content of nickel particles, indicating catalytic effect of nickel particles. During the carbonization of PAN, it has been confirmed that PAN provided drastic structural changes at the carbonization stages: extended thread-like, rod-like and spherulitic structures. Interestingly, the carbon prepared by heat treatment at $800^{\circ} \mathrm{C}$ showed the thread-like structure extended from a nickel particles or the aggregation, and the car- bon at $1000^{\circ} \mathrm{C}$ showed the rod-like structure whose one end side was closed by a nickel particle or the aggregation. These structural changes indicated the catalytic effect of nickel particles on the carbonization. The X-ray analysis revealed that the structure was composed of the two kinds of crystal carbon structure (G- and T-components) and amorphous carbon regions. At $1600^{\circ} \mathrm{C}$, the diffraction peak became sharper with decreasing lattice fluctuation of the lattice distance between the (002) planes, and the diffraction peak top was almost equal to the top reported for perfect graphite. To pursue the detailed analysis of the turbostratic structures, the X-ray diffraction intensity curve was analyzed in terms of the concept concerning para-crystalline theory of Hoseman and Bagchi. As the result, it turned out that the broad intensity peak at $c a .26 .5^{\circ}$ was attributed to small size of the G-component with very small lattice fluctuation (almost zero) between the (002) planes. On the other hand, the T-component was found to be composed of unstable small structures with very large lattice fluctuation.

\section{REFERENCES}

1. R. Setnescu, S. Jipa, T. Setnescu, W. Kappel, and Z. Osawa, Carbon, 37, 1 (1999).

2. T. Ohnish, I. Murase, T. Noguchi, and M. Hirooka, Synth. Met., 18, 497 (1987).

3. Y. Kaburaki and Y. Hishiyama, Carbon, 33, 773 (1995).

4. C. W. Jones and W. J. Koros, Carbon, 32, 1419 (1994).

5. T. A. Centeno and A. B. Fuertes, J. Membr. Sci., 160, 201 (1994).

6. H. Konno, R. Matsuura, M. Yamasaki, and H. Habazaki, Synth. Met., 125, 167 (2002).

7. Y. Kaburagi, H. Hatori, A. Yoshida, Y. Hishiyama, and M. Inagaki, Synth. Met., 125, 171 (2002).

8. M. Inagaki, K. Fujita, Y. Takeuchi, K. Oshida, H. Iwata, and H. Konno, Carbon, 39, 921 (2001).

9. M. Scheffler, P. Greil, A. Berger, E. Pippel, and J. Woltersdorf, Mater. Chem. Phys., 84, 131 (2004).

10. J. Libera and Y. Gogotsi, Carbon, 39, 1307 (2001).

11. A. Oya, Tanso, 102, 118 (1980).

12. A. Oya, M. Mochizuki, S. Otani, and I. Tomizuka, Carbon, 17, 71 (1979).

13. H. Hatori, S. Hishiki, T. Kobayashi, Y. Yamada, T. Nishio, and M. Shiraishi, Tanso, 189, 165 (1999).

14. N. A. Kiselev, J. Sloan, D. N. Zakharov, E. F. Kukovitskii, J. L. Hutchison, J. Hammer, and A. S. Kotosonov, Carbon, 36, 1149 (1998).

15. Y. Z. Bin, K. Oishi, A. Koganemaru, D. Zhu, and M. Matsuo, Carbon, 43, 1617 (2005).

16. M. Kruk, B. Dufour, E. B. Celer, T. Kowalewski, M. Jaroniec, and K. Matyjaszewski, J. Phys. Chem. B, 109, 9216 (2005).

17. P. R. Giunta, L. J. van de Burgt, and A. E. Stiegman, Chem. Mater., 17, 1234 (2005). 
18. R. Ryoo, S. H. Joo, and S. J. Jun, J. Phys. Chem. B, 103, 7743 (1999).

19. H. Q. Hou and D. H. Reneker, Adv. Mater., 16, 69 (2004).

20. S. H. Park, S. M. Jo, D. Y. Kim, W. S. Lee, and B. C. Kim, Synth. Met., 150, 265 (2005).

21. A. B. Fuertes, G. Marban, and J. Munniz, Carbon, 34, 223 (1996).

22. W. Zhang, J. Liu, and G. Wu, Carbon, 41, 2805 (2003).

23. D. Zhu, C. Y. Xu, N. Nakura, and M. Matsuo, Carbon, 40, 363 (2002).

24. D. Zhu, A. Koganemar, C. Xu, Q. Shen, S. Li, and M. Matsuo, J. Appl. Polym. Sci., 87, 2063 (2003).

25. J. J. Ge, H. Hou, Q. Li, M. J. Graham, A. Greiner, D. H. Reneker, F. W. Harris, and S. Z. D. Cheng, J. Am. Chem. Soc., 126, 15754 (2004).

26. B. Hoseman and S. N. Bagchi, "Direct analysis of diffraction by matter.” Amsterdam, North-Holland, 1962.

27. R. Lamber, N. Jaeger, and G. Schulz-Ekloff, Surf. Sci., 197, 402 (1988).

28. S. Otani, A. Oya, and J. Akagami, Carbon, 13, 353 (1975).

29. J. B. Donnet, T. K. Wang, and J. C. M. Peng, "Carbon fiber," 3rd ed., Marcel Dekker, New York, 1998.

30. N. Iwashita, C. R. Park, H. Fujimoto, H. Shiraishi, and M. Inagaki, Carbon, 42, 701 (2004).

31. M. Inagaki, H. Mugishima, and K. Hosakawa, Tanso, 74, 76 (1973).

32. M. Kaburagi, Y. Bin, D. Zhu, C. Xu, and M. Matsuo, Carbon, 42, 915 (2003).

33. M. Matsuo and C. Kitayama, Polym. J., 17, 479 (1985).

34. M. Matsuo, M. Tamada, T. Terada, C. Sawatari, and M. Niwa, Macromolecules, 10, 988 (1982). 